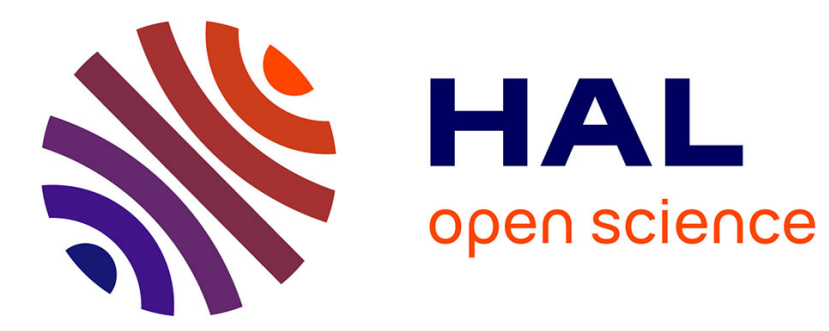

\title{
Un contrat agraire à Lucques au début du IXe siècle
} Anne Mailloux

\section{To cite this version:}

Anne Mailloux. Un contrat agraire à Lucques au début du IXe siècle: Concession d'une terre et d'une maison en Lucchesia (802). Histoire et sociétés rurales, 1996, 6, pp.117-125. halshs-00505149

\section{HAL Id: halshs-00505149 \\ https://shs.hal.science/halshs-00505149}

Submitted on 19 Jan 2022

HAL is a multi-disciplinary open access archive for the deposit and dissemination of scientific research documents, whether they are published or not. The documents may come from teaching and research institutions in France or abroad, or from public or private research centers.
L'archive ouverte pluridisciplinaire HAL, est destinée au dépôt et à la diffusion de documents scientifiques de niveau recherche, publiés ou non, émanant des établissements d'enseignement et de recherche français ou étrangers, des laboratoires publics ou privés. 


\section{SOURCES}

\section{UN CONTRAT AGRAIRE À LUCQUES AU DÉBUT DU IXe SIÈCLE}

Anne MAILLOUX*

$\mathbf{L}$

ES ACTES DE LUCQUES DES VIII-XX SIĖCLES - quelque deux mille pièces aux archives archiépiscopales 1 - ont été édités à la fin des années 1830 par Domenico Bertini et Domenico Barsocchini, dans la série des Memorie e Documenti per servire all'istoria del Ducato di Lucca ${ }^{2}$. Ce travail considérable est malheureusement dépourvu d'appareil critique. Des erreurs et des lacunes récurrentes y sont aussi repérables, différentes $d^{\prime}$ un auteur à l'autre: Barsocchini abrégeait les descriptions de biens, très standardisées, par des points entre parenthèses, ou par la mention «ecc», considérant qu'elles n'étaient qu'un ornement; il ne transcrivait pas non plus les qualités des témoins, rejoint en cela par Bertini qui taisait jusqu'à leur nom. En 1'absence d'édition scientifique postérieure ${ }^{3}$, des vérifications systématiques s'imposent donc, qui sont aussi l'occasion de dénicher quelques actes passés jusquelà inaperçus. Plusieurs mises à jour ont déjà été effectuées et quelques textes signalés, voire édités 4 . Ayant eu à mon tour la bonne fortune de retrouver un contrat agraire inédit, quoique déjà daté et classé, je le propose à l'attention des lecteurs d'Histoire et Sociétés Rurales.

L'étude des contrats agraires est d'une importance primordiale pour l'histoire sociale, économique et politique du haut Moyen Âge. Elle est souvent rendue impossible par leur absence même : les sources les plus fréquemment utilisées pour déterminer les rapports liant les propriétaires fonciers et les « tenanciers » chargés de la mise en valeur de l'espace agricole sont les polyptyques, inventaires des biens, personnes et redevances établis par les grands propriétaires dans un but de rationalisation de la gestion de leur domaine 5 .

* École française de Rome, Palazzo Farnese, Piazza Farnese 67, 00186 Roma.

Je tiens à remercier Giuseppe Ghilarducci, le conservateur de 1'Archivio Arcivescovile de Lucca - qu'on citera désormais AAL-, ainsi que Graziano Concioni, de m'avoir accueillie chaleureusement à l'Archivio, et d'avoir autorisé cette publication.

1. Quelques documents sont conservés à l'Archivio di Stato di Lucca, mais en nombre très limité.

2. Cités $M D$.

3. SCHIAPARELLI, 1929-1933, ne reprend les actes privés que jusqu'à la chute du royaume lombard, en 774; les Chartae Latinae Antiquiores (citées ChLA) s'arrêtent en 800.

4. GIUSTI, 1973; WICKHAM, 1978; EGGER, 1992.

5. TOUBERT, 1983; FOSSIER, 1978. La nature de ces documents constitue donc un filtrage très net de la réalité que nous tentons ici d'appréhender. 
L'aire italienne fait figure d'exception à cette période, car la tradition écrite y est plus vivace, $d^{\prime}$ une part, et les fonds d'archives plus anciens et mieux conservés.

Les contrats agraires, qui formalisent les rapports économiques, sociaux et juridiques entre un propriétaire foncier et un «locataire-tenancier» chargé de l'entretien et de la mise en valeur des biens concédés, permettent d'analyser, presque au microscope (nous réfléchissons ici à l'échelle de l'exploitation agricole, voire de la parcelle), les modalités, la chronologie et la tonalité de ces liens. D'autre part, ils fournissent des renseignements précis sur ce qui est leur premier objet, les modes de mise en valeur de l'espace agricole : types de culture, redevances, rythmes, répartition des productions selon les caractéristiques morphologiques de l'espace, densité de l'habitat.

La définition de ces rapports est l'enjeu d'un débat historiographique : il $s^{\prime}$ agit dans un premier temps d'avoir une vision plus nette des modalités d'émergence d'un système d'exploitation agraire désormais bien connu - celui de la curtis carolingienne, ou grand domaine -, mais aussi de corriger cette approche (qui n'entrevoit pas, pour l'époque carolingienne, d'alternative à ce système), liée à la monophonie des sources les plus généralement disponibles (les polyptyques cités plus haut). En aval, l'étude des contrats agraires renseigne sur les évolutions de la fin de l'époque carolingienne, qui sont politiques certes, mais aussi sociales et économiques : c'est dans ce cadre (celui d'une approche sociale, juridique et économique) que les contrats agraires apportent des éléments plus précis, en tout cas plus circonstanciés, sur l'émergence du système seigneurial.

Dans ce contexte, le fonds de l'Archivio Arcivescovile de Lucques constitue pour le haut Moyen Âge un ensemble particulièrement intéressant, tant par la quantité que par la qualité de conservation et la variété des chartes. Il est formé essentiellement d'actes de la pratique privée, qui fournissent une masse de renseignements de tous ordres pour la connaissance de la société du haut Moyen Âge dans cette région. On peut se livrer à une analyse des pratiques notariales, mais aussi des transactions foncières, des relations économiques et sociales entre propriétaires et locataires, des types de dépendance induits par ces transactions. Il est ainsi possible d'avoir une idée assez précise des modes de mise en valeur des terres, mais aussi de la forme des parcellaires et des paysages agraires.

Plusieurs auteurs se sont employés, dans des perspectives diverses, à analyser ces sources : Hansmartin Schwarzmaier ${ }^{6}$ fut le premier à avoir une approche assez complète de ces archives, les utilisant essentiellement pour éclaircir les liens sociaux et les dynamiques politiques qui animent la cité de Lucques au cours du haut Moyen Âge. Il met en place une prosopographie des familles puissantes de Lucques et des différents «cercles» actifs de la cité : les ducs puis les comtes, les évêques et les membres de l'église lucquoise, les notaires sont autant de groupes et de fonctions qu'il suit avec pré-

6. SCHWARZMAIER, 1972 et 1973. 
cision et dont il souligne le degré d'intégration dans l'Empire. Les approches de Bruno Andreolli ${ }^{7}$ et de Chris Wickham ${ }^{8}$ sont légèrement différentes : tous deux se livrent à une analyse économique et sociale à l'échelle du territoire de Lucques, le premier dans une perspective $d^{\prime}$ histoire rurale et agraire, le second dans l'idée de définir les systèmes des dépendances et leurs dynamiques, avec pour terme l'émergence de nouvelles formes socio-politiques au XIe siècle.

Le texte qui suit est une concession agraire de 802, exprimée sous la forme d'un engagement prononcé par le preneur. On peut le désigner comme livello, selon la terminologie courante en Italie ${ }^{9}$ : contrat agraire passé avec des locataires cultivateurs du lot de terres qui leur est assigné. Alors que ce type d'acte ne concerne que $13 \%$ du fonds lucquois au vine siècle, il en représente plus de la moitié au siècle suivant; entre les années 750-800 et 801-850, le nombre des concessions est multiplié par six ${ }^{10}$. L'augmentation est suffisamment importante pour laisser penser qu'elle n'est pas fortuite et correspond véritablement à une évolution des structures foncières, sociales, économiques, mais aussi des modes de mise en valeur de l'espace agraire.

Certains éléments sont récurrents dans ces contrats: obligation de résidence sur le fonds, «amélioration » de la terre et de l'immeuble, participation à l'exercice de la justice domaniale - clause absente ici-, prestations sur la pars dominica. Pour mieux évaluer le contenu de notre concession, on peut la comparer à une autre, de 809 , déjà éditée11, dans laquelle le même bailleur, Alprandus recteur de l'église Saint-Pierre de Vigisimo, concède une autre maison, dite massaricia, et des biens à un autre tenancier. Les deux textes diffèrent sur presque tous les points. Le statut de la maison (massaricia) dans le second va de pair avec une position différente du tenancier, dont la dépendance personnelle envers le bailleur est plus marquée que celle qui lie l'homme libre «ordinaire ». Cette dépendance est renforcée par l'emploi d'un autre formulaire pour introduire la concession : les mots repromitto et manum meam facio tibi, par lesquels s'engage le preneur, expriment une soumission plus forte, dont on trouve l'écho dans le reste du texte. L'obligation de se présenter devant Alprandus, sur sa convocation - ad mandato vestro venire : devant le tribunal domanial, ou pour exécuter un service de courrier? -, est ainsi spécifiée. La peine encourue en cas de non-respect des clauses est fixée à 100 sous, contre 30 dans le premier cas - une somme raisonnable, et cou-

7. ANDREOLLI, 1978a et $\mathrm{b}, 1983$.

8. WiCKHAM, 1980, 1988, 1995.

9. Livello, en écho à la formule souvent présente selon laquelle les contrats sont passés livellario nomine. Pour la mise au point terminologique, et une définition précise de ce type de concession, cf. ANDREOLLi, 1978a, p. 69-70. Chris Wickham donne aussi des éléments de réflexion à ce sujet (WICKHAM, 1978 et 1985).

10. Bien entendu, ce type d'évaluation n'est que le reflet des sources disponibles. Malgré tout, l'abondance relative des sources lucquoises permet d'utiliser ce type d'appréciation à titre indicatif.

11. AAL 382 (éd. $M D, v / 2, n^{\circ} 366$ ), octobre 809, Lucques (acte vérifié par mes soins). Le texte est aussi analysé par ANDREOLLI, 1978a. 
rante dans la Lucchesia à cette époque. Enfin, le régime de l'angaria paraît plus strict : il dépend de la seule volonté d'Alprandus, udque (sic) tibi utilitas fuerit, et non d'une coutume antérieure négociée, en vertu de laquelle l'angaria n'est due dans le premier texte qu'à Vigisimo, à l'exclusion de tout autre lieu. La différence de statut entre les deux tenanciers, ou plutôt entre les deux pièces de terre concédées, se conjugue peut-être avec la nécessaire adaptation $\mathrm{du}$ formulaire que provoque la mise en place progressive du régime domanial, où le poids des prestations en travail est l'élément porteur et nécessaire de la relation contractuelle et du régime de mise en valeur des biens du propriétaire. En contrepartie, il n'est pas question de redevance en nature (forfaitaire ou proportionnelle à la récolte) ou en argent dans le deuxième contrat ${ }^{12}$.

La définition de l'angaria est problématique, car le terme correspond à des réalités mouvantes : de l'Antiquité romaine, où il désigne une institution publique, service de transport ou de manutention, au haut Moyen Âge, il glisse vers des formes de prestations de nature et de statut différents, devenant une charge à caractère privé, consistant parfois en une autre prestation que celle d'un service de transport, assimilée parfois à une marque de servitude. On traduit souvent angaria par corvée ${ }^{13}$, mais il semble que cette acception directe doive être validée avec beaucoup de précautions. À Lucques notamment, on éprouve une grande difficulté à établir cette assimilation pour l'époque lombarde et carolingienne, même si l'angaria, dans les contrats, est toujours placée dans les clauses relatives aux prestations pesant sur les paysans. Elle ne dénote pas forcément un statut servile ${ }^{14}$ car la plupart des concessionnaires sont libres, qualité qui, au demeurant, conditionne leur capacité à contracter. Elle ne sous-entend pas explicitement un travail agraire : la formulation des contrats est souvent trop vague pour l'affirmer, en tout cas pour l'époque qui nous concerne, où l'on se réfère à la coutume sans en donner le contenu. Elle ne peut être non plus un service de transport, dans la zone lucquoise : quand celui-ci est exigé, il est explicité clairement ${ }^{15}$. Dans un cas comme dans l'autre, l'expression angaria medietas, indicative $\mathrm{d}^{\prime}$ une fréquence (trois jours par semaine) laisse penser qu'il s'agit d'une prestation en travail, mais rien ne permet d'affirmer pleinement que celui-ci est agricole. Cette précision de la fréquence est une nouveauté par rapport aux

12. La redevance est évoquée, de manière assez vague, dans le premier texte, par les termes usus et consuetudo. L'absence de précision est plutôt inhabituelle dans le contexte lucquois, où la spécification de ce type de prestations, avec un certain luxe de détail, et une variété des exigences, semble une caractéristique notable ; cf. le tableau analytique établi par ANDREOLLI, 1978a.

13. Pour une approche terminologique: NIERMEYER, Jan Frederik, Mediae Latinitatis Lexicon minus, Leyde, E. J. Brill, 1976, s. v., p. 43. Voir aussi les analyses d'Andreolli, qui établit directement la corrélation entre angaria et corvée (ANDREOLLI, 1978 et 1987).

14. ANDREOLLI, 1987.

15. Ainsi, dans un autre acte de 802 , un concessionnaire se voit imposer deux fois par an un transport depuis Cecina, à $30 \mathrm{~km}$ au sud de Lucques, jusqu'à la ville (AAL n ${ }^{\circ} 316$, éd. $M D, \mathrm{v} / 2$, $n^{\circ} 303,30$ octobre $802,+\mathrm{H} 76$; acte vérifié par mes soins). 
actes du vmi siècle. Son succès à partir des années 830 est un autre indice de l'insistance sur le travail propre au régime domanial, qui a concouru à niveler par le bas le statut du tenancier.

Pour autant, on se gardera de parler ici de « grand domaine », car les deux actes, établis pour l'exploitation des biens dépendants d'une modeste église rurale, ne correspondent qu'aux besoins d'une petite propriété. L'insistance sur l'angaria pourrait s'y expliquer par la nécessité qu'éprouve le recteur de l'église de faire cultiver les terres par des dépendants logés.

Une ultime remarque concerne la durée de validité du contrat : si la plupart des livelli comportent généralement une indication de durée limitée, de 29 ans le plus souvent, qui en fait des baux emphytéotiques, à Lucques cette précision quantifiée de la durée est rare ; tout au plus peut-on trouver des formules laissant ouverte la possibilité de la transmission du bien par le tenancier à ses fils (ego cum heredibus meis). Cette dernière particularité renforce l'originalité de l'espace lucquois en matière contractuelle : on y observe un usage et une utilisation «à géométrie variable» du stock de formules, avec parfois création de formules spécifiques, qu'on peut étudier dans le détail sur la durée de deux ou trois siècles. Dans le cas présent, 1'absence de mention de durée laisse supposer que le bien sera de nouveau attribué par le recteur de Saint-Pierre de Vigisimo à la mort du tenancier. Sa gestion, prudente, veille à ne pas laisser de possibilités d'aliénation indirecte ou implicite de ses biens par les tenanciers. Cette idée est renforcée par le fait qu'il n'y a pas de clause de sécurité mettant en question la responsabilité de l'église vis-à-vis du locataire - en cas de rupture du contrat par imposition de charges supplémentaires, ou en obligeant le tenancier à évacuer les biens -, ce qui est le cas dans d'autres actes lucquois.

L'étude de ce contrat est donc d'un double intérêt. D'une part, il est «exemplaire» d'une situation sociale, économique et juridique générale, commune à beaucoup d'espaces italiens : la manière de formaliser et d'affirmer les rapports entre propriétaires de la terre et tenanciers répond à des canons, des formules, des chemins qui renvoient aux réalités du monde carolingien en pleine transformation en ce début du IXe siècle. Il permet d'autre part de faire le point sur les particularités de l'espace lucquois et sur la spécificité du cas étudié ici : de petites unités d'exploitation, mais aussi de petites ou de moyennes propriétés, dont on peut entrevoir le mode de fonctionnement, en marge du modèle classique du «grand domaine».

\section{Bibliographie}

\section{Sources}

MD : Memorie e Documenti per servire all'istoria del ducato di Lucca, Atti della Reale Accademia Lucchese, t. IV / 1 et 2, éd. Bertini, Domenico, Lucques, 1818-1836 (réimpr. anastatique: 1994); t. v/2 et 3, éd. Barsocchini, Domenico, Lucques, 1837-1841 (réimpr. anastatique : 1971).

SCHIAPARELLI, Luigi, (éd.), Codice Diplomatico Longobardo, 2 vol., Rome, Istituto storico italiano per il medio evo, 1929-1933 (Fonti per la storia d'Italia, 62-63), xIV-386 p. et 476 p.

Manaresı, Cesare, (éd.), I Placiti del «Regnum Italiae», t. I, Rome, Istituto storico italiano per il medio evo, 1955 (Fonti per la storia d'Italia, 92). 
GIUSTI, Martino, «Documenti lucchesi anteriori al secolo IX : supplementi all'edizione Bertini et Barsocchini», in Lucca e la Tuscia nell'alto medioevo. Atti del $V^{\circ}$ Congresso internazionale di studi sull'alto medioevo (Lucca, 3-7 ott. 1971), Spolète, 1973, p. 703-707.

WICKHAM, Chris, "Due carte inedite lucchesi dell'ottavo secolo", Bollettino Storico Pisano, 67, 1978, p. 185-193.

ChLA : BRUCKNER, Albert, et MARICHAL, Robert, (éd.), Chartae Latinae Antiquiores, vol. XXX à XL, Dietikon-Zürich, Urs Graf, 1988-1991.

EGGER, Christopher, «Eine neuaufgefundene Privaturkunde des 10. Jahrhunderts aus Lucca», Mitteilungen des Instituts für österreichische Geschichtsforschung, 100, 1992, p. 150-160.

\section{Études}

ANDREOLLI, Bruno, «Contratti agrari e patti colonici nella Lucchesia dei secoli vII e IX», Studi medievali, 3e s., 19, 1978a, p. 69-158;

- «Ad conquestum faciendum. Un contributo per lo studio dei contratti agrari altomedievali », Rivista di storia dell'agricoltura, 18, 1978b, p. 109-136;

-, «L'evoluzione dei patti colonici nella Toscana dei secoli VIII-IX», Quaderni medievali, 16, 1983, p. 29-52;

-, « La forza del diritto : lo ius libellarium e le sue variazioni durante il Medioevo », in Le campagne italiane prima e dopo il Mille, Bologne, CLUEB, 1985 (Biblioteca di storia agraria medievale, 2), p. 275-309;

-, «La corvée precarolingia», in Le prestazioni d'opera nelle campagne italiane del Medioevo, IX Convegno Storico di Bagni di Lucca (1-2 Giugno 1984), Bologne, CLuEB, 1987 (Biblioteca di storia agraria medievale, 3), p. 13-33.

Fossier, Robert, Polyptyques et censiers, Turnhout, Brepols, 1978, 72 p. (Typologie des sources $d u$ Moyen Âge occidental, 28-A. rv. 1).

PetRucCl, Armando, "Il Codice n. 490 della Biblioteca capitolare di Lucca», Actum Luce, 2, 1973, p. 159-175 (repris et modifié in PETRUCC, Armando, et ROMEO, Carlo, "Scriptores in urbibus», in Alfabetismo e cultura scritta nell'Italia altomedievale, Bologne, il Mulino, 1992, chapitre 4).

SChIAPARELLI, Luigi, Il Codice 490 della Biblioteca Capitolare di Lucca e la scuola scrittoria lucchese (sec. VIII-IX). Contributi allo studio della minuscola precarolina in Italia, Rome, Biblioteca Vaticana, 1924 (Studi e Testi, 36), 116 p.

SCHWARZMAIER, Hansmartin, Lucca und das Reich bis zum Ende des 11. Jahrhunderts, Studien zur Sozialstruktur einer Herzogstadt in der Toskana, Tübingen, M. Niemeyer, 1972 (Bibliothek des Deutschen Historischen Instituts in Rom, 41), xII-450 p.;

-, "La società lucchese nell'alto medioevo e gli archivi ecclesiastici di Lucca", in Lucca archivistica, storica, economica. Relazioni e comunicazioni al XV Congresso Nazionale Archivistico (Lucca, ottobre 1973), Rome, 1973 (Fonti e Studi del Corpus membranarum italicarum, 10), p. 175-191.

SUPINO MARTINI, Paola, «Le sottoscrizioni testimoniali al documento italiano del secolo vIII : le carte di Lucca», Bullettino dell'Istituto Storico Italiano per il Medio Evo, 98, 1992, p. 87-108.

TOUBERT, Pierre, «Il sistema curtense : la produzione e lo scambio interno in Italia nei secoli VIII, IX e X», in Storia d'Italia. Annali, 6 : economia naturale, economia monetaria, Turin, Einaudi, 1983, p. 5-63 (repris dans Histoire du haut Moyen Âge et de l'Italie médiévale, Londres, Variorum Reprints, 1987, art. VII).

WICKHAM, Chris J., «Economic and social institutions in Northern Toscana in the 8th. century», in Istituzioni ecclesiastiche della Toscana medioevale, Galatina, Congedo Ed., 1980 (Studi e ricerche [della] Commissione italiana per la storia delle pievi e delle parrocchie, 1), p. 7-34;

-, The Mountains and the City : the Tuscan Appennines in the Early Middle Ages, Oxford, Clarendon Press, 430 p.;

-, Comunità e clientele nella Toscana del XII secolo. Le origini del comune rurale nella Piana di Lucca, Rome, Viella, 1995, 287 p. 
28.

\section{Octobre 802 \\ Concession d'une terre et d'une maison en Lucchesia}

Source : A. Archivio Arcivescovile di Lucca, Diplomatico, n 317, ++ B 33.

Iohannes fils de feu Teudipertus promet à Alprandus prêtre, fils de feu Auriprandus, de résider et d'habiter dans la maison qui dépend de l'église SaintPierre à Vigisimo ${ }^{1}$, où réside Austripertulus; il doit mettre en valeur les biens attribués et s'engage à s'acquitter des redevances coutumières et de l'angaria, à peine de 30 sous.

Notes dorsales : dans le sens du texte au recto, au milieu, au crayon, « $317 » ; « 800$. $B n^{\circ} 33$. Joannes vendr bona Valprando »; " 803 »; presqu'effacé, $d^{\prime}$ une main du XIeXII siècle : «+ De casa et re Sancti Petri de vice I simo»; en dessous, " ++B n 33 ».

Le prêtre Rachiprandus, rédacteur du document, apparaît de manière récurrente dans les sources lucquoises : il écrit pour la première fois un acte en 770 comme simple clerc ${ }^{2}$, est qualifié de prêtre à partir de 779 , porte le titre de lociservator de l'évêque dans deux jugements en 785-7863. Actif jusqu'en 809 , il a laissé 24 documents de sa main, sans compter les nombreuses chartes qu'il a souscrites. Personnage important du milieu notarial lucquois, il a aussi un rôle majeur dans la curie épiscopale : sa fonction de lociservator («lieutenant») dans les deux jugements cités peut en témoigner, mais c'est surtout l'expression Rachiprandus presbiter notarius Sancte Ecclesie qui l'atteste : il est ainsi placé aux côtés du magister scole cantorum au sommet de la hiérarchie de l'administration lucquoise ${ }^{4}$.

Son écriture, définie soit comme une "minuscule précaroline» (ScHIAPARELLI, 1924), soit comme une «cursive nouvelle italienne» (PETRUCCI, 1973), permet de le placer dans la lignée des principaux scribes du vIII siècle, héritier de son concitoyen Gaudentius, notaire et maître de l'école de Lucques. Sa main est enfin reconnue dans un des manuscrits les plus fameux de la bibliothèque du chapitre (ms 490), une collection de textes antiques et du haut Moyen Âge, où il a probablement copié la deuxième partie du Liber Pontificalis.

1. L'église Saint-Pierre de Vigisimo peut être identifiée soit à San Pietro a Cappiano, soit à San Pietro a Castelfranco di Sotto, deux localités situées dans le Valdarno inferiore, entre le canal majeur de l'Usciana et l'Arno, à peu de distance de Fucecchio : REPETT, v, p. 767. Dizionario geographico-fisico storico della Toscana contenente la descrizione di tutti i luoghi del Granducaton Ducato di Lucca, Garfagnana e Lunigiana, t. 5, Florence, 1846, p. 767. Au haut Moyen Âge, cette zone, au sud du marais de Fucecchio, fait partie du diocèse de Lucques, à sa périphérie.

2. AAL 125 (éd. $M D, \mathrm{v} / 2, \mathrm{n}^{\circ} 124$ et $C h L A, \mathrm{n}^{\circ} 1017$ ), 23 septembre 770 , Tempagnano.

3. Ibid., 212 et 220 (éd. MD, v/2, n 202 et 211; MANARESI, 1955, n 6-7; ChLA, nos 1098 et 1106).

4. Ibid., 381 (éd. $M D, v / 2, \mathrm{n}^{\circ} 365$ ), 21 septembre 809 , Lucques (acte vérifié par mes soins). 


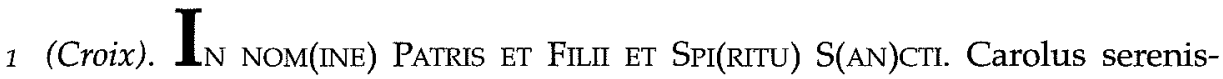
simus augustus et $\mid$ a $\mathrm{D}(\mathrm{eo})$ coronatus magnus et pacificus imperator romanum gubernans 1 imperium qui et per misericordiam $D(e i)$ rex Francor(um) et Langobardolrum, anno regni eius quo Langob(ar)diam 5 coepit vigisimo nono I et filius eius domino nostro Pippino rege, anno regni eius vigisimo secundo $\mathrm{m}(\mathrm{ense}) \mid$ octubris indictione decima ${ }^{\mathrm{a}}$. Manifestum est mihi Iohan I nes fili(u)s q(uon)d(am) Teudip(er)ti quia p(er) hanc cartulam repromitto tibi Alprande pr(es)b(ite)r I filio b(one) $m$ (emorie) Auriprandi ut ego resedere et abitare debeam in casa $\mid$ et res tua illa $q(u e)$ est $p(e r)$ tene $<n>s$ de ecclesia vestra sancti Petri in Loco Vigisimo I ubi residde(t) Austrip(er)tulus et tam ipsa casa quam et omnia res ad sup(er) I tenentes ego bene lavorare et gubernare seu meliorare debeam et $\mathrm{p}(\mathrm{er}) \mid$ singul $<0>\mathrm{s}$ ann $<0>\mathrm{s}$ ego tibi facere et adimplere promitto usum et consuetudi I ne ipsis case sic(ut) exinde consuetudo fuit; angaria autem medietaltem tibi p(er) singulos annos facere debeam, in s(upra)s(crip)to loco vigisimo tantum. I Et si a me hec omnia s(upra)s(crip)ta capitula per singulos annos sic non fue I rint adimpleta et conservata qualiter hic superius legitur I vel si ego foris de pred(ic)ta casa et res exire presumpsero alibi ad abiltand(i) tunc spondeo me $q(u i)$ $\mathrm{s}$ (upra) Iohannes esse componitur(u)s tibi Alprando I pr(es)b(iter)o sol(idos)

20 triginta, quia tal(iter) inter nos convenit, et Rachiprandum I pr(es)b(iteru)m scribere rogavi. Actum Luca.

Sign(um) (croix) $\mathrm{m}(\mathrm{anu}) \mathrm{s}$ Iohannis qui hanc cartulam fieri rogavit.

Sign(um) (croix) m(anu)s Ilmerandi filius Silvuli cl(ericus) testis.

(Croix) Ego Ghisimundus pr(es)b(iter) rogatus a Iohanne me teste subs(cripsi) .

(Croix) Ego Teutprandus cl(ericus) rogatus a Iohanne me teste subs(cripsi) ${ }^{b}$.

(Croix) Ego Rachiprandus pr(es)b(ite)r post tradita complevi et dedi.

a. Undecima serait plus juste pour la cohérence de la datation

b. Souscription autographe ; sur la question, cf. SUPINO MARTINI, 1992. 


\section{Traduction}

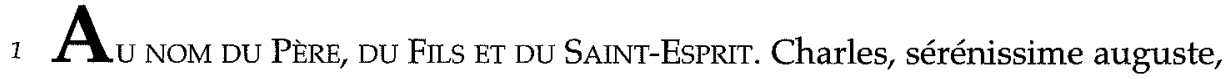
couronné par Dieu grand et pacifique empereur des Romains, gouvernant l'empire et, par la miséricorde de Dieu, roi des Francs et des Lombards, vingt-neuvième année de son règne depuis qu'il a pris la Lombardie, et vingt-deuxième année de son fils, notre seigneur Pépin, roi, au mois d'octobre, onzième indiction. Moi, Jean, fils de feu Teudipertus, déclare promettre par cette charte à toi Alprandus, prêtre, fils d'Auriprandus, de bonne mémoire, de résider et d'habiter dans ta maison et tes biens, qui dépendent de votre église Saint-Pierre au lieu Vigisimo, où réside Austripertulus. Je devrai mettre en valeur, gérer et améliorer aussi bien la maison que tous les biens qui en dépendent et je promets de faire et de verser chaque année l'usage et la coutume de cette maison, selon la coutume jusqu'ici ; je devrai par ailleurs te faire chaque année la moitié de l'angaria au lieu susdit Vigisimo. Et si toutes les clauses que dessus ne sont pas accomplies et observées par moi chaque année, ainsi qu'il est écrit plus haut, ou si je tente de quitter cette maison et ces biens pour habiter ailleurs, alors je m'engage, moi, Jean, à te payer, Alprandus prêtre, de trente sous, car tel est convenu entre nous, et j'ai demandé à Rachiprandus, prêtre, d'écrire [l'acte]. Fait à Lucques.

Seing (croix) de la main de Jean,

qui a demandé que soit faite cette charte.

Seing (croix) de la main d'Ilmerandus, fils de Silvulus, clerc, témoin.

(Croix) Moi, Ghisimundus, prêtre, requis par Jean, j'ai souscrit comme témoin.

(Croix) Moi, Teutprandus, clerc, requis par Jean, j'ai souscrit comme témoin.

(Croix) Moi, Rachiprandus, prêtre, après la transaction, j'ai complété et donné [1'acte]. 